\title{
Structure and function of fusion pores in exocytosis and ectoplasmic membrane fusion
}

\author{
Manfred Lindau and Wolfhard Almers
}

\author{
Max Planck Institut für medizinische Forschung, Heidelberg, Germany
}

\begin{abstract}
Several proteins involved in exocytosis have been identified recently, but it is still completely unclear which molecules perform the fusion event itself. Although in viral fusion the fusion proteins are known, even there the molecular mechanism remains controversial. Investigation of single fusion events by electrophysiological techniques together with fluorimetric measurements have now provided some insight into the properties of the first aqueous connection, the fusion pore. This pore has an initial size similar to an ion channel and allows movement of lipids only after it has substantially expanded, indicating that it is initially not a purely lipidic structure, but incorporates lipids when it expands. Although neurotransmitter release may occur through narrow transient fusion pores, the fusion pore of synaptic vesicles probably expands very rapidly, making it unlikely that secretion is performed by rapid exo/endocytosis without full fusion under normal conditions. Recent recordings from small membrane patches have made it possible to resolve fusion events from vesicles as small as synaptic vesicles. Future experiments using excised patches may provide an approach

to identify the molecular machinery of exocytotic membrane fusion.
\end{abstract}

Current Opinion in Cell Biology 1995, 7:509-517

\section{Introduction}

Exocytosis of a secretory vesicle requires a large number of proteins acting in a probably well defined sequence that is still incompletely known $[1,2]$. The last and most mysterious step in this sequence is the fusion of the vesicle membrane with the plasma membrane. During fusion, an aqueous connection forms between the vesicle lumen and the extracellular space. This connection is initially no wider than a large ion channel, and has been termed the fusion pore $[3,4]$. Here, we review the properties of fusion pores and their physiological consequences, and then discuss the molecular nature of early fusion pores in the light of new findings.

All present knowledge about fusion pores comes from physical measurements during single fusion events. Exocytosis of a single secretory granule causes a stepwise increase in the electrical capacitance of the plasma membrane $[5,6]$, occurring when the fusion pore opens and the vesicle adds its membrane to the cell surface. This is most easily seen in cells with large secretory granules, such as rat mast cells $(0.8 \mu \mathrm{m}$ granule diameter; [6]), mast cells from 'beige' mice, a mutant with unusually large secretory granules $(1-5 \mu \mathrm{m} ;[3,7])$, and eosinophils $(0.5-2 \mu \mathrm{m} ;[8,9])$. Very recently, it has also become possible to make such observations during the exocytosis of small vesicles, some as small as synaptic vesicles $\left[10^{\bullet \bullet}\right]$. One surprising finding has emerged in all studies: upward steps in membrane capacitance are very occasionally followed by downward steps of the same amplitude, as if the fusion pore of a secretory granule opened and then closed again ('capacitance flicker'). This was also observed when fusion was mediated by influenza virus hemagglutinin [11]. Apparently, at least early steps in fusion are reversible.

With large secretory vesicles, an even earlier sign of fusion can be detected electrically. A brief and transient electric current flows as the vesicle discharges its membrane potential through the nascent fusion pore $[4,12,13]$. Analysis of such electric discharges allows one to determine the electrical conductance of the fusion pore over the first milliseconds of its existence [12]. The conductance time course showed two kinetic components. First the conductance increased abruptly, much as the conductance of an ion channel increases when the channel opens in a patch clamp experiment. Next, the conductance increased gradually, as if the pore expanded. In mast cells of beige mice the initial expansion rate is about $200 \mathrm{pS} \mathrm{ms}^{-1}$, suggesting that the pore diameter doubles in $6 \mathrm{~ms}$ at $23^{\circ} \mathrm{C}$. It was proposed that the abrupt opening of the fusion pore represents a protein conformational change similar to the opening of an ion channel whereas the subsequent, gradual expansion reports the recruitment of lipid into the pore circumference ([14]; see below). 
Initial fusion pore conductances are compiled in Table 1 for different cells; the table also includes the initial conductance of the pore formed during cell fusion mediated by the fusion-catalyzing envelope proteins of the influenza and Semliki forest viruses. In each system, the initial pore conductance is highly variable. The mean values, however, fall within a factor of three, and are similar to those of large ion channels $\left(\mathrm{Ca}^{2+}\right.$-activated $\mathrm{K}^{+}$channels, 100-200 pS; voltage-gated anion channels of mitochondria, $400 \mathrm{pS}$; [15]). Evidently the initial aperture of a fusion pore is no larger than that of a large ion channel.

Table 1. Initial fusion pore conductances.

\begin{tabular}{|c|c|c|c|c|}
\hline & Method & $\begin{array}{l}\text { Peak of } \\
\text { distribution }\end{array}$ & Mean & Reference \\
\hline $\begin{array}{l}\text { Mouse mast } \\
\text { cell }\end{array}$ & Transient* & $200 \mathrm{pS}$ & $330 \mathrm{pS}$ & [12] \\
\hline $\begin{array}{l}\text { Horse } \\
\text { eosinophils }\end{array}$ & Transient* & $100 \mathrm{pS}$ & $200 \mathrm{pS}$ & [13] \\
\hline \multicolumn{4}{|l|}{ Human } & {$\left[10^{\bullet \bullet}\right]$} \\
\hline Influenza $\mathrm{HA}$ & Transient* & ND & $150 \mathrm{pS}$ & [11] \\
\hline $\begin{array}{l}\text { Semliki forest } \\
\text { virus }\end{array}$ & dc current ${ }^{\ddagger}$ & $300 \mathrm{ps}$ & ND & [64] \\
\hline $\begin{array}{l}\text { *Transient: el } \\
\text { the moment } \\
\text { ‡dc current: } \\
\text { cells. ND, no }\end{array}$ & $\begin{array}{l}\text { rical discharge } \\
\text { usion pore op } \\
\text { asured by a d } \\
\text { etermined. }\end{array}$ & $\begin{array}{l}\text { e of the vesic } \\
\text { oning. }{ }^{\dagger} \text { Adm } \\
\text { ouble voltage }\end{array}$ & $\begin{array}{l}\text { e membr } \\
\text { ttance: se } \\
\text { clamp of }\end{array}$ & $\begin{array}{l}\text { capacitance at } \\
\text { fusing insect }\end{array}$ \\
\hline
\end{tabular}

\section{Slow expansion of the fusion pore can retard the release of transmitter or hormone}

In the first $10-20 \mathrm{~ms}$, fusion pores increase their conductance tenfold or more, but then the expansion process slows markedly $[4,13,16]$. The pore conductance often changes little for several seconds, and sometimes the pore even closes again (capacitance flicker). Pores of conductances as large as $8 \mathrm{nS}$ have been seen to close [12]; such a pore has an estimated diameter of $20 \mathrm{~nm}$ and would be readily visible under an electron microscope.

What obstacle might temporarily slow the expansion of fusion pores? The obstacle must be at least partly cytosolic, because cytosolic $\left[\mathrm{Ca}^{2+}\right]$ increases the rate of expansion of large pores in eosinophils [13] and mast cells [17]. It would be of interest whether this also applies to neurons and neuroendocrine cells, wherein exocytosis is triggered by a large increase in subplasmalemmal $\left[\mathrm{Ca}^{2+}\right]$. In eosinophils, cytosolic $\left[\mathrm{Ca}^{2+}\right]$ influences neither the initial pore conductance nor the initial, rapid expansion phase ([13]; our unpublished data).

The slow expansion of fusion pores delays the moment where vesicle and plasma membrane merge completely, and slows the release of the vesicle contents. Nonetheless, transmitters or hormones leak out well before fusion is complete, as is apparent from the time course of release from single granules (Fig. $1 \mathrm{a}-\mathrm{c}$ ). In cases where the secreted substance has a redox potential less than that of water, this time course has been monitored by 'amperometric' recording with a carbon microelectrode placed next to a single cell [18]; the microelectrode records the current generated when the transmitter is oxidized or reduced. Although most of the granulc contents are released synchronously, this explosive release is often preceded by periods where transmitter leaves the vesicle slowly (indicated by arrows in Fig. 1). This was found in all three cell types where recordings of this type have been made. In mast cells, granules have been seen to open their fusion pore, release part of their contents, and close again [19]. Cytosolic $\left[\mathrm{Ca}^{2+}\right]$ in mast cells shortens the period of slow transmitter release and hastens the moment of synchronous discharge, as it facilitates the expansion of the fusion pore [17].

Evidently the small size of the fusion pore delays the release of material from large dense-core secretory granules (Fig. 1a-c). This creates no problem with hormones that act slowly, for example, through $G$ proteins or signaling cascades. At a synapse, however, speed is of the essence and synaptic vesicles must release their transmitter in fractions of a millisecond. Part of this challenge is met by keeping synaptic vesicles small, smaller than any other membranous organelle. Thus diffusion distances remain short, and vesicles may release more than $60 \%$ of their contents in $260 \mu$ s even if a $300 \mathrm{pS}$ pore does not dilate at all [20]. It has been suggested, however, that an open vesicle releases transmitter very much faster, and that this could not be explained by simple diffusion [21].

Even in synaptic vesicles, transmitter occasionally escapes slowly, as if hindered by a narrow and/or slowly expanding fusion pore. The most striking recordings come from the electric organ of Torpedo with its slightly larger (80-100 nm diameter) synaptic vesicles (Fig. 1d). The majority of vesicles open rapidly, but around $15 \%$ seem to open more slowly, with an initial period of slow transmitter release similar to that seen in most amperometric recordings from dense-core vesicles. This observation suggests that normal synaptic vesicles have mechanisms to hasten the escape of transmitter, but that this mechanism sometimes fails.

It has been proposed $[22,23]$ that synaptic vesicles may open their fusion pore, let transmitter out, close the pore again and be re-loaded with transmitter without ever fusing into the plasma membrane. The speed and precision of membrane retrieval in an endocrine cell suggests that this may also be possible with dense-core vesicles [24]. This mechanism, sometimes called 'kiss-and-run', avoids 
(a)

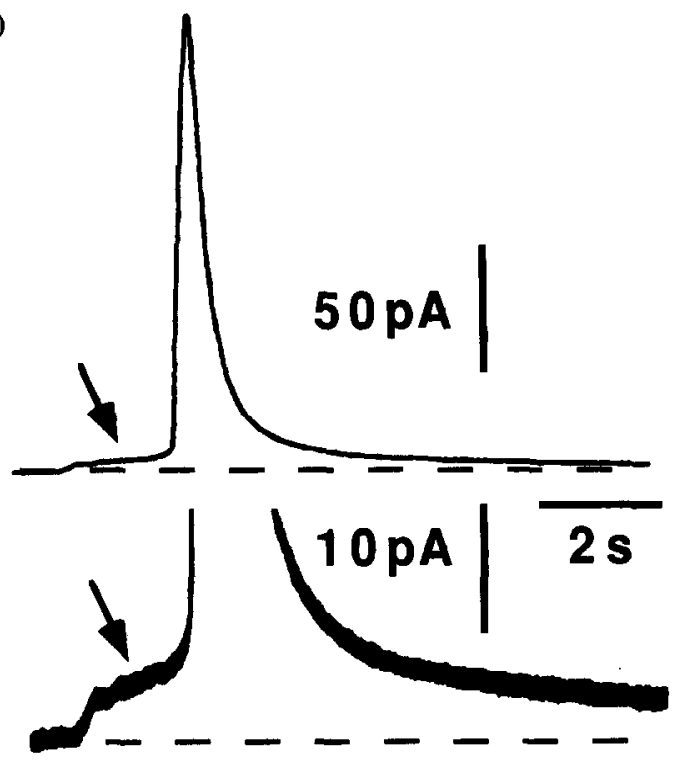

(c)

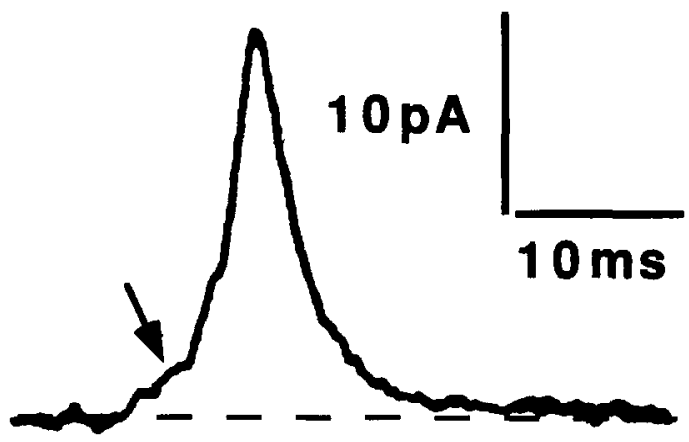

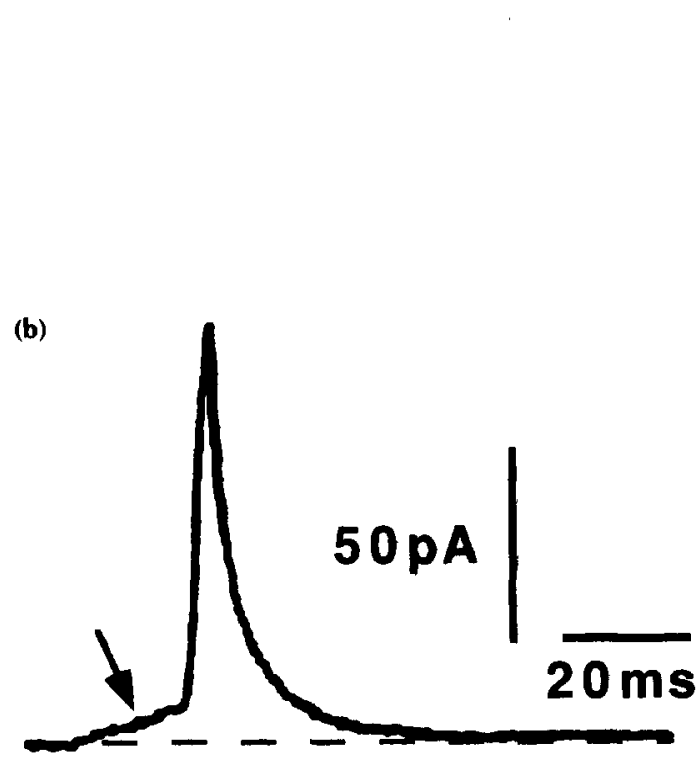

(d)

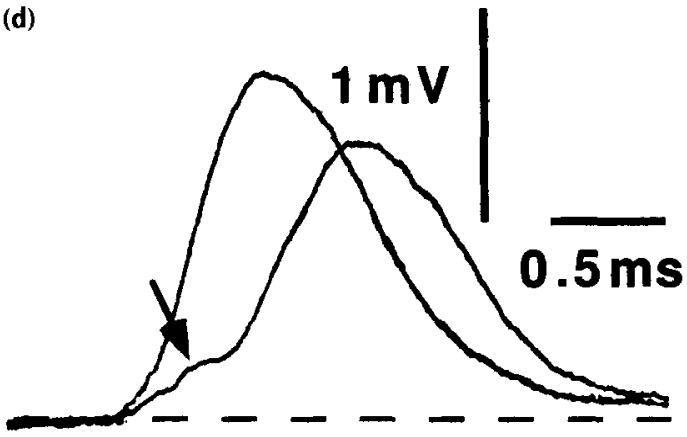

Fig. 1. Exocytic release from single granules and vesicles detected as amperometric signals (a-c) or as miniature end plate currents (d). The large deflections represent the nearly synchronous release occurring after the fusion pore has become wide, or the granule/vesicle has merged into the plasma membrane. They are preceded by a small 'foot' (arrow) representing slow release through a fusion pore which has not yet expanded fully. (a) Release of serotonin from a beige mouse mast cell granule (diameter $2.8 \mu \mathrm{m}$ ). The lower panel is a portion of the upper trace shown on an expanded ordinate (from [19]). (b) Release of serotonin from a chromaffin granule (diameter 280-300 $\mathrm{nm}$; from [59]). (c) Release of dopamine from a glomus cell of the carotid body (granule diameter $\sim 120 \mathrm{~nm}$; from [60]). (d) Release of acetylcholine causes two miniature postsynaptic potentials from the electric organ of Torpedo marmorata (vesicle size $\sim 80 \mathrm{~nm}$; from [61]). One rises rapidly and represents the majority, the other shows an initial 'foot' similar to those seen with amperometry; this type of waveform was observed in about $15 \%$ of the recordings. These potentials were recorded with an extracellular loose-patch pipette; the signals are proportional to the inward current flowing through acetylcholine receptor channels.

the molecular recognition events needed to selectively retrieve vesicle membrane proteins that have diffused into the plasma membrane. Although occasional or even repeated cycles of kiss-and-run seem possible and physiologically useful, three findings indicate that the 'kiss' is ultimately terminal and full fusion with the plasma membrane inevitable. First, after intense cell stimulation, vesicle membrane proteins appear on the cell surface $[25,26]$. Second, in a Drosophila mutant shibire, with its defect in the protein dynamin $[27,28]$, clathrin-coated vesicles cannot separate from the cell surface and motor synapses rapidly run out of synaptic vesicles [29]. Finally, although transient pore openings do release serotonin from mast cell granules [19], they are always followed by irreversible fusion.

\section{Fission pores and endocytosis}

When exocytosed membranc is retrieved, vesicles bud from the plasma membrane and then pinch off. In posterior pituitary nerve terminals stimulated exhaustively by high cytosolic $\left[\mathrm{Ca}^{2+}\right]$, large vacuoles pinch off the cell surface, a process that can be followed electrophysiologically. For hundreds of milliseconds, the vacuole lumen remains connected to the external 
medium by an aqueous connection (the fission pore) whose conductance declines exponentially until it is too small to be measured $(<40 \mathrm{pS})$ [30]. What causes these endocytic vesicles to pinch off from the plasma membrane? The dynamin GTPase has been shown to wind around the openings of clathrin-coated vesicles, and it may be part of the molecular machinery that pinches these vesicles off the plasma membrane $\left(\left[31^{\bullet \bullet}, 32^{\bullet \bullet}\right]\right.$; see also Lamaze and Schmid, this issue, pp 573-580). Perhaps dynamin also has a more general role in pinching off vacuoles and vesicles that are not clathrin-coated. It would be of interest if dynamin is already present at exocytic docking sites before vesicles undergo exocytosis; if so, the closing of an expanded fusion pore perhaps uses a molecule such as dynamin.

\section{The mechanism of membrane fusion}

How biological membranes fuse remains unknown. To facilitate discussion, we sort the hypotheses advanced to date into two broad groups. The first group, which we will call 'proximity models', holds that proteins fuse lipid bilayers by bringing them into close contact; once close enough, bilayers fuse on their own. A proximity model in pure form is that of Monck and Fernandez [33] wherein a 'scaffold' of unknown motor proteins is said to pull the bilayers of the fusing compartments together. A more explicit model of this type is the 'stalk model' [34] shown in Figure 2b, wherein a known fusion-catalyzing protein, the influenza virus hemagglutinin (HA), engages two apposed bilayers using hydrophobic peptides and pulls them together. In all proximity models, fusion proceeds via 'hemifusion' (Fig. 2a), an intermediate state where the apposed leaflets from each of two bilayers have merged and their lipids mix, but where the remaining two leaflets have formed a new hybrid bilayer that separates the compartments. This bilayer must later be pierced to form an aqueous connection (not shown in Figs 2a,b). An attraction of such models is that pure lipid bilayers, if composed of the right lipids, may indeed be fused in this way, and do perform hemifusion [35]. Furthermore, in endocrine cells phosphatidylinositol-4,5-bisphosphate must be generated before $\mathrm{Ca}^{2+}$ can trigger exocytosis $\left[36^{\circ} \cdot\right.$. This finding highlights the importance of lipids at an early step in exocytotic membrane fusion.

In the second class of hypotheses, the fusion pore models, the gap between the fusing membranes is bridged by a

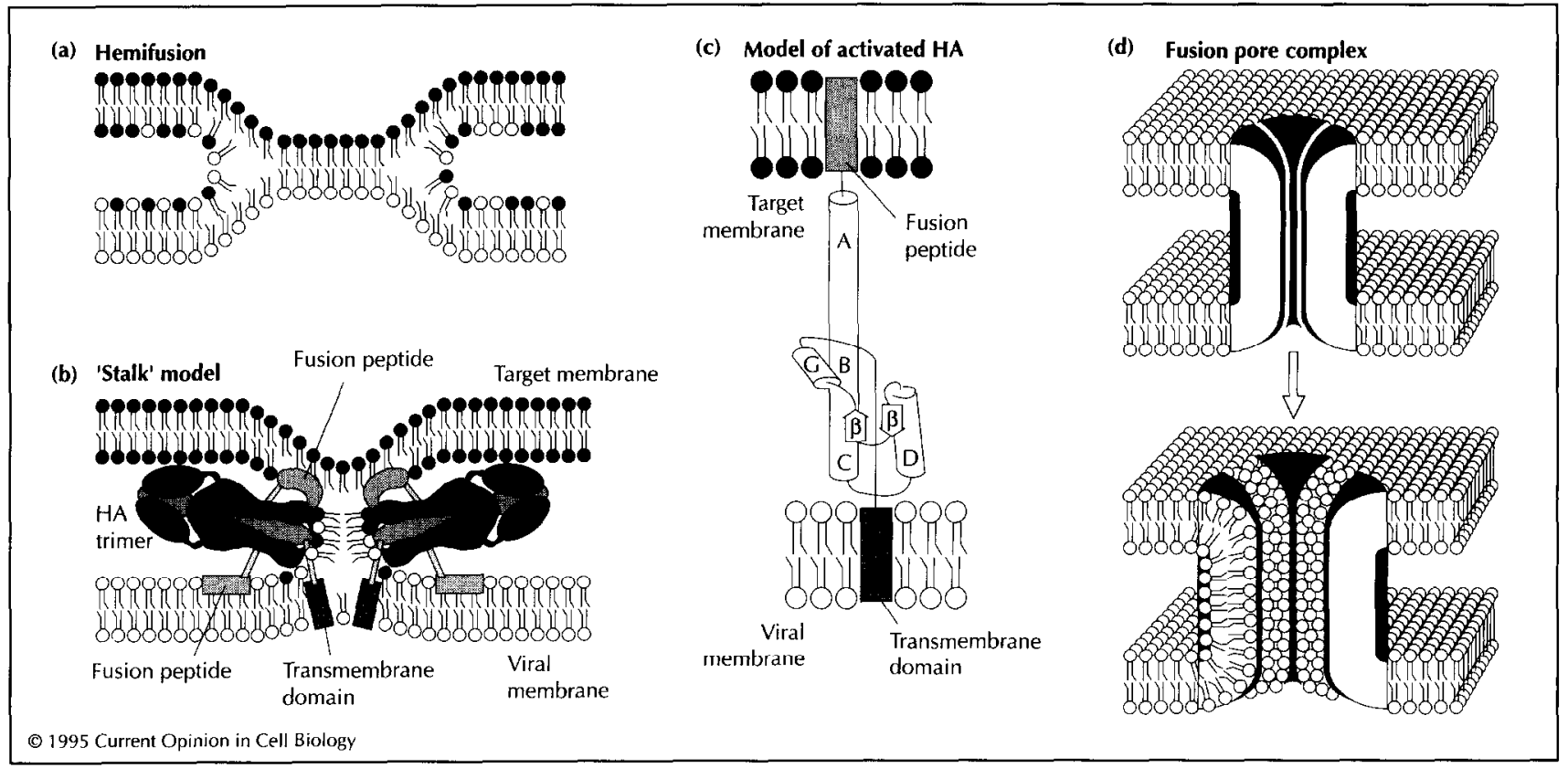

Fig. 2. Possible mechanisms of membrane fusion. (a) In hemifusion, each of two bilayers fuse one of their membrane leaflets; the lipids in the two leaflets mix but an aqueous pore connecting the lumen of the fusing compartments is not formed. (b) In the 'stalk' model of fusion, activation of influenza HA causes the hydrophobic fusion peptides of this molecule to swing out near where HA emerges from the viral membrane. The molecule must tilt on its side to allow the fusion peptides to engage both the target and viral membranes. (The HA trimer is shown in silhouette.) Pulling the membranes together causes their outer leaflets to fuse, creating the stalk as an intermediate. This model attributes no role to the transmembrane domains of $\mathrm{HA}$ and does not take into account new X-ray structural data. (Modified after [34,49,62]). (c) Transient conformation of active influenza HA inferred from X-ray crystallography [54 ${ }^{\bullet \bullet}$ ]. For simplicity, only a single monomer is shown, and the sialic acid binding portion of the molecule is omitted. The fusion peptide and transmembrane domain are at opposite ends of the molecule. As in (d), $\mathrm{HA}$ forms a protcinaceous bridge inserting into both the viral and target bilayers; in this conformation, transmembrane domain and fusion peptide could serve analogous and essential roles in fusion. The disposition of $\alpha$-helices (labeled A-D and G) and $\beta$-sheets (B) are from the X-ray structure of Bullough et $\mathrm{al}$. . [54*0]. As the fusion peptide and transmembrane domain were absent in the crystal, their disposition is hypothetical. In particular, there is no evidence that the fusion peptide sparis the target membrane. (Modified after Hughson [63].) (d) Fusion by a hypothetical fusion pore macromolecule. In the top part, the pore complex has just formed an aqueous channel. In the bottom part, the subunits of the structure have moved apart, and lipid molecules have diffused into the interstices (modified from [20]). 
hypothetical protein complex whose opposite ends enter and/or span both bilayers (Fig. 2d). The first version of this model was proposed by Pfenninger et al. [37]. A more recently model views the complex as a radially symmetric structure made of subunits $[14,20]$. Upon activation, the complex undergoes a conformational change so that an aqueous channel connects the two fusing compartments, much like the way a gap junction channel connects two cells (Fig. $2 \mathrm{~d}$, top). The subunits then move apart radially and expose amphipathic surfaces between them that allow the inward migration of lipids (Fig. $2 \mathrm{~d}$, bottom). In this way the pore can expand indefinitely. Another possible representative of this class is presented in [38]; interesting hybrids with proximity models have been proposed by White [39] and Bentz et al. [40]. Fusion pore models have two attractive features. (i) In exocytosis, large protein oligomers are known to assemble between fusing membranes before catalyzing fusion; they would tend to oppose proximity but might well form aqueous channels. (ii) $\mathrm{Ca}^{2+}$-gated ion channels are known to be gated open and shut in small fractions of a millisecond, so the model makes it easy to understand the enormous speed of exocytosis during synaptic transmission.

The following electrophysiological observations are consistent with fusion pore models. (i) Both the generally abrupt opening of fusion pores and their initial conductances are clearly reminiscent of ion channels. Admittedly, fusion pores sometimes open gradually, but such gradual openings, although unusual for ion channels, are regularly observed when gap junction channels open for the first time [41]. (ii) The pore expansion rate increases steeply with temperature (3-4-fold over $10^{\circ} \mathrm{C}$; [12]). This is not expected if lipids invade the lining of the pore by diffusing past other lipid molecules, because the diffusion coefficient of lipids in lipid bilayers changes only weakly with temperature unless there is a phase transition [42]. Either lipid molecules must bind to and unbind from proteins as they invade the pore circumference, or the early expansion phase is caused by a protein conformational change. In either case, the steep temperature-dependence argues against the initial pore being purely lipidic.

Some electrophysical observations have been taken to argue against the fusion pore model. (i) When the membrane surrounding an isolated secretory granule was ruptured beneath a patch pipette [43], the initial conductance of the resulting hole was $1120 \pm 0.5 \mathrm{pS}$ $(n=3)$, a value felt to be similar to those in Table 1 . As it seemed unlikely that the patch pipette opened a genuine fusion pore (or the vesicle's half of a fusion pore), this 'breakdown pore' was attributed to a hole in the lipid bilayer. Furthermore, breakdown pores occasionally closed, as do fusion pores. The authors concluded [43] that the electrophysiological behavior of fusion pores can be explained by a single lipid bilayer, as would occur during hemifusion, and does not require the pore to be proteinaceous. (ii) An elegant study of capacitance flicker [44] showed that expanded pores allow the flux of membrane from the cell surface into the vesicle before they close. Although this finding says little about the properties of the initial pore, it does show clearly that expanded pores are predominantly made from fluid lipid bilayer, as predicted by all models. A critical role for the lipid 4,5-phosphoinositide is suggested by the requirement for inositide phosphorylation before $\mathrm{Ca}^{2+}$-triggered exocytosis in an endocrine cell [36*0].

\section{Structure and function of a viral envelope fusion-catalyzing protein}

In exocytosis, the molecular nature of the fusion-catalyzing complex is essentially unknown. Two synaptic vesicle proteins, VAMP/synaptobrevin [45] and synaptophysin [46] have been suggested to be intimately involved in fusion, but direct evidence is lacking. The situation is more favorable for fusion mediated by identified viral envelope proteins [47]. These envelope proteins catalyze 'ectoplasmic fusion' where the extracellular leaflets are closest. Their mechanism may differ from that of 'endoplasmic fusion' where the cytosolic leaflets encounter each other first, as in exocytosis.

Viral envelopes are populated at high density with membrane proteins that catalyze their fusion with a host cell membrane. The protein of the influenza virus, a homotrimer termed HA, has been extensively characterized. Each monomer spans the viral membrane once, and the structure of its large extraviral domain has been established by $\mathrm{X}$-ray diffraction [48].

HA and other viral fusion proteins carry a 16-23 amino acid long hydrophobic sequence called the fusion peptide that is essential for fusion [47]. In the quiescent HA trimer, the fusion peptide is buried within the protein and inaccessible to antibodies. However, when HA is activated by the acidic environment of the endosome, it assumes a short-lived fusogenic conformation that inactivates rapidly and irreversibly. The fusion peptide swings out, becoming accessible to antibodies, and enters whatever lipid bilayer is closest [49-51]. There it probably stays indefinitely, being the only part of the protein that becomes significantly labeled when HA interacts with bilayers containing a photoactivatable lipid [52]. The labeling pattern suggests that the fusion peptide forms an $\alpha$-helix in the bilayer; its 23 amino acids (for example, [47]) make it long enough to span it.

In the quiescent $\mathrm{HA}$ at neutral $\mathrm{pH}$, the fusion peptides seem to be in the wrong place to interact with the target membrane. Buried close to where HA emerges from the viral bilayer, they are a full $10 \mathrm{~nm}$ away from the tip of the molecule, where HA carries sialic acid binding sites (one per monomer) attaching it to gangliosides in the target membrane. Because of the location of the fusion peptide, some models have viewed HA as lying on its side while mediating fusion (Fig. 2b).

A major advance was made when Carr and Kim [53] noticed that the fusion peptide was attached to a peptide with an amino acid sequence typical for the 


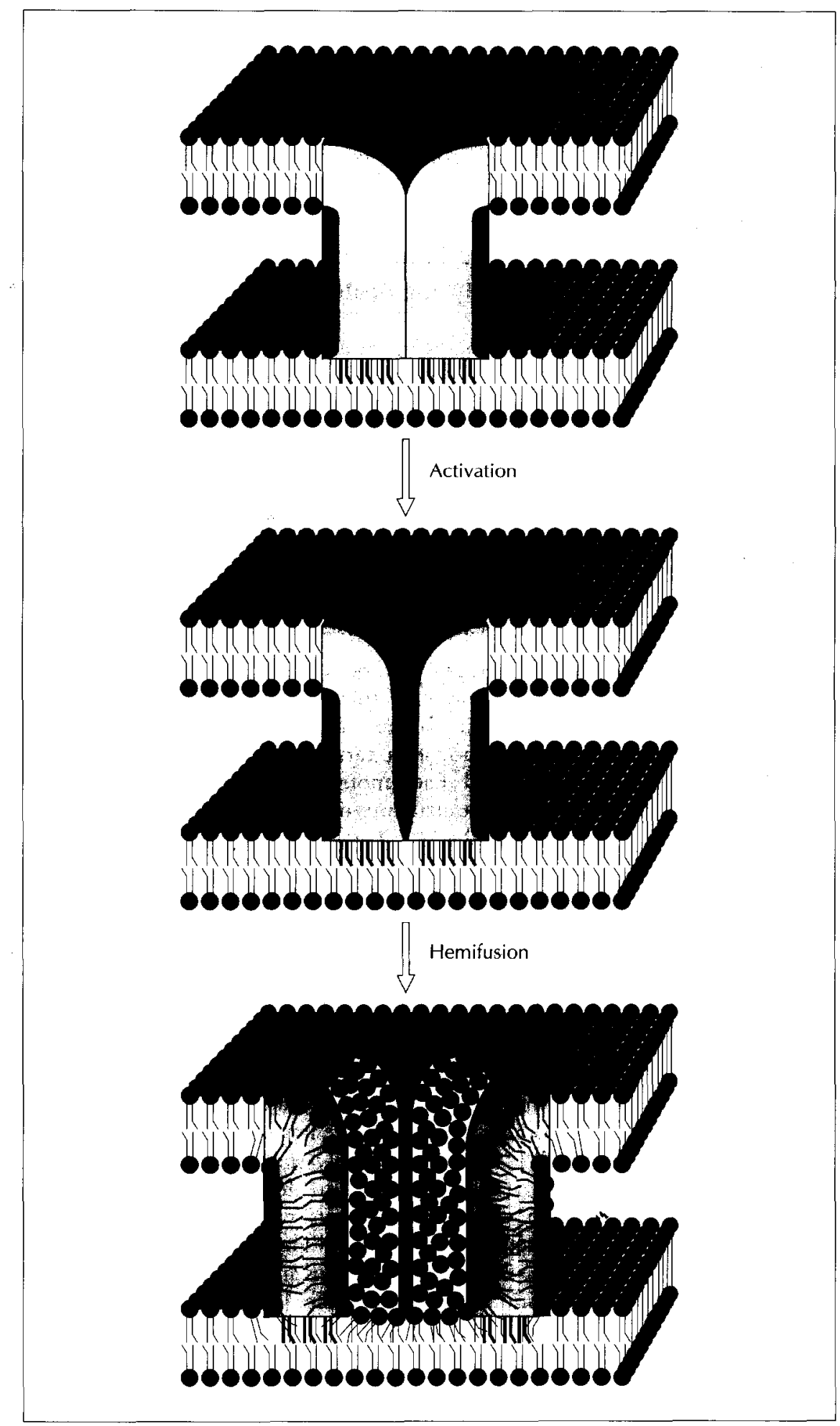

Fig. 3. Modification of the model shown in Fig. $2 d$ to account for hemifusion after deletion of a transmembrane domain. The fusion pore complex is made up of several subunits with hydrophilic surfaces (green) and lipophilic surfaces (yellow). In one membrane (e.g. the viral membrane; light blue) the transmembrane domains were replaced with GPI anchors (in red), as in the experiment of Kemble et al. [58*0]. Note that in the middle panel, the fusion pore ends blind where transmembrane domains are absent (compare with Fig. 2d). In the lower panel, the incomplete pore has expanded, its subunits have dissociated, and lipids have diffused into the interstices along the exposed amphipathic surfaces. This has led to mixing of lipids in the apposed leaflets of the two bilayers. Where normally an aqueous connection would have penetrated the viral membrane (Fig. 2d; [20]), hemifusion has occurred and a bilayer has formed.

coiled-coil interaction of $\alpha$-helices. In the quiescent trimer, this 'attachment peptide' forms a helical hairpin rather than a coiled coil. When it was synthesized and acidified, however, it gave a circular dichroism signal consistent with coiled coils. The authors suggested that on acidification, the attachment peptide straightens its hairpin, forms a three-stranded coiled coil with its sisters in the other two monomers, jack-knifes out of the molecule and carries the fusion peptide to the very tip of the molecule, where it can insert into the target membrane. Recently, Bullough et al. [54०0] solved the $\mathrm{X}$-ray structure of a large $\mathrm{HA}$ fragment at low $\mathrm{pH}$ that includes the attachment peptide. A coiled coil was indeed found, and the new structure is consistent with the fusion peptide swinging to the tip of the HA protein (Fig. 2c). 
Despite our extensive knowledge about the structure of influenza HA, the mechanism of fusion catalysis by this molecule remains unclear. Does HA mediate fusion by proximity? In Figure 2c, the fusion protein has entered and/or spans both bilayers with its opposite ends, yet the bilayers remain separated by a $15 \mathrm{~nm}$ long proteinaceous bridge. A similar topology may well exist in exocytic fusion, where a large molecular fusion complex (20S complex; [55]) is thought to assemble between vesicle and plasma membrane.

Some recent experiments have begun to test the two classes of model more directly.

\section{Lipid mixing versus aqueous connection}

In proximity models, hemifusion allows lipid mixing before an aqueous connection is formed between the fusing compartments, whereas in fusion pore models, the aqueous connection forms first, and lipid mixing occurs only once the pore has expanded and become partially lipidic. To test these models, the outer membrane leaflet of erythrocytes (red blood cells) was labeled with a fluorescent lipid, and the erythrocyte was attached to a fibroblast expressing HA on its cell surface. Fusion of the cell pair was triggered by acidification, and the diffusion of lipid from the erythrocyte into the fibroblast was monitored by quantitative image analysis. Simultaneous electrical measurements showed that a fusion pore opened minutes before the first signs of lipid transfer [56,57]. In fact, lipid transfer appeared to require a threshold pore conductance of $500 \mathrm{pS}$ [56]. The experiment argues against hemifusion as an intermediate.

\section{The transmembrane domain of HA is essential for fusion}

Fusion pore models, but not proximity models, require that the fusion complex inserts into both fusing membranes. Recently, Kemble et al. [58*0] made an HA mutant replacing the transmembrane region with a glycophosphatidylinositol (GPI) anchor. Activation of the mutant caused hemifusion but no aqueous connection. The result was taken to confirm a critical prediction of proximity models, namely that hemifusion is a fusion intermediate [58.0]. In contrast, we view the requirement for entry of the protein into both membranes as support for the fusion pore model.

The finding seemingly contradicts the result with intact HA [56]; however, an incomplete fusion pore protein (Fig. 3) could also cause hemifusion. The fusion pore would open and expand in the bilayer where the protein bears a membrane-inserting domain (the fusion peptide). On the other side where such an element is missing, however, the aqueous channel cannot form and ends blind (compare Fig. 2d with Fig. 3). When the subunits of the structure disperse, inward migration of lipid could create a bilayer where there would normally have been an aqueous connection (Fig. 3, lower panel).

In our opinion, the GPI-anchored HA mutant rules out fusion by proximity alone. Hemifusion is the closest conceivable proximity, yet an aqueous connection or fusion pore is not formed.

\section{Conclusions}

Exocytosis starts with the abrupt opening of a narrow fusion pore, whose conductance (and hence diameter) is no larger than that of a large ion channel. The fusion pore expands, at least ultimately, by recruiting lipid into its circumference. Full expansion takes time and can retard the release of contents from dense-core secretory granules and even synaptic vesicles. Future work must address the molecular structure of the initial fusion pore. Already it is clear, however, that its behavior is inconsistent with a purely lipidic structure. Most likely, the initial fusion pore is surrounded by a ring of protein, and it expands by breaking the ring and recruiting lipid into its circumference.

To identify the molecular machinery performing the exocytotic fusion event, development of a reconstituted system will be instrumental. The recent demonstration of exocytotic events in cell-attached patches [10** suggests that it may also become possible to use excised patches where the composition on the cytoplasmic side can be readily controlled. This approach may provide the potential to disintegrate the cellular fusion machinery to a point where fusion is inhibited, thus reducing the system from a state where all required components are present (as is the case in an intact cell) to a system where necessary components may be removed or inactivated. Such a system has the great advantage that the individual fusion event can be studied at very high resolution. Recording of individual fusion pore openings makes it possible to determine if the fusion process studied has the same or at least similar properties to that of the exocytotic event in the intact cell. Reconstitution of fusion pores with the same properties as in the natural systems from purified lipids and proteins may eventually provide the ultimate proof for identification of the molccular machinc(s) performing exocytotic fusion events.

\section{References and recommended reading}

Papers of particular interest, published within the annual period of review, have been highlighted as:

- of special interest

-• of outstanding interest

1. Jahn R, Südhof TC: Synaptic vesicles and exocytosis. Annu Rev Neurosci 1994, 17:219-246.

2. Bajjalieh $\mathrm{SM}$, Scheller $\mathrm{RH}$ : The biochemistry of neurotransmitter secretion. J Biol Chem 1995, 270:1971-1974.

3. Breckenridge LJ, Almers W: Final steps in exocytosis observed in a cell with giant secretory granules. Proc Natl Acad Sci USA 1987, 84:1945-1949.

4. Breckenridge LJ, Almers W: Currents through the fusion pore that forms during exocytosis of a secretory vesicle. Nature 1987, 328:814-817.

5. Neher E, Marty A: Discrete changes of cell membrane capacitance observed under conditions of enhanced secretion 
in bovine adrenal chromaffin cells. Proc Natl Acad Sci USA 1982, 79:6712-6716.

6. Fernandez IM, Neher E, Comperts BT: Capacitance measurements reveal stepwise fusion events in degranulating mast cells. Nature 1984, 312:453-455.

7. Zimmerberg J, Curran M, Cohen FS, Brodwick M: Simultaneous electrical and optical measurements show that membrane fusion precedes secretory granule swelling during exocytosis of beige mouse mast cells. Pror Natl Acad Sci USA 1987. 84:1585-1589.

8. Lindau $M$, Nüe $O$, Bennett J, Cromwell $O$ : The membrane fusion events in degranulating guinea pig eosinophils. / Cell Sci 1993, 104:203-209.

9. Scepek S, Lindau L: Focal exocytosis by eosinophils compound exocytosis and cumulative fusion. EMBO / 1993, 12:1811-1817.

10. Lollike $K$, Borregaard $N$, Lindau $M$ : The exocytotic fusion pore - of small granules has a conductance similar to an ion channel. I Cell Biol 1995, 129:99-104.

By cell-attached patch-clamp recording in human neutrophils, the resolution was improved to allow recording the exocytosis of vesicles as small as $60 \mathrm{~nm}$ as a capacitance step. The recordings were made with an $8 \mathrm{kHz}$ sinusoid; this frequency is too high to allow significant gating of voltage-gated ion channels within one sinusoidal period. For $200 \mathrm{~nm}$ vesicles, it was possible to record the conductance of the fusion pore (see Table 1). The approach should allow future recording of the exocytosis of single synaptic vesicles.

11. Spruce AE, Iwata A, Almers $W$ : The first milliseconds of the pore formed by a fusogenic viral envelope protein during membrane fusion. Proc Natl Acad Sci USA 1991, 88:3623-3627.

12. Spruce AE, Breckenridge LJ, Lee AK, Almers W: Properties of the fusion pore that forms during exocytosis of a mast cell secretory vesicle. Neuron 1990, 4:643-654.

13. Hartmann J, Lindau $M$ : A novel $\mathrm{Ca}^{2+}$ dependent step in exocytosis subsequent to vesicle fusion. FEBS Lett 1995, 363:217-220.

14. Almers W: Exocytosis. Annu Rev Physiol 1990, 52:607-624.

15. Hille B: Ionic channels of excitable membranes. Sunderland, MA: Sinauer Associates, Inc: 1992.

16. Curran MJ, Cohen FS, Chandler DE, Munson PJ, Zimmerberg J: Exocytotic fusion pores exhibit semi-stable states. I Membr Biol 1993, 133:61-75.

17. Fernández-Chacón R, Alvarez de Toledo G: Cytosolic calcium facilitates release of secretory products after exocytotic vesicle fusion. FEBS Lett 1995, 363:221-225.

18. Whightmann RM, Jankowski JA, Kennedy RT, Kawagoe KT, Schroeder Tl, Leszczyszyn DI, Near JA, Diliberto El, Viveros $\mathrm{OH}$ : Temporally resolved catecholamine spikes correspond to single vesicle release from individual chromaffin cells. Proc Natl Acad Sci USA 1991, 88:10754-10758.

19. Alvarez de Toledo G, Fernández-Chacón $R$, Fernandez JM: Release of secretory vesicle products during Iransient vesicle fusion. Nature 1993, 363:554-558.

20. Almers W, Tse FW: Transmitter release from synapses: does a preassembled fusion pore initiate exocytosis? Neuron 1990 , $4.813-818$

21. Khanin $R$, Parnas $H$, Segel L: Diffusion cannot govern the discharge of neurotransmitter in fast synapses. Biophys / 1994, 67:966-972.

22. Meldolesi ), Ceccarelli B: Exocytosis and membrane recycling. Philos Trans $R$ Soc Lond Biol 1981, 296:55-65.

23. Fesce $R$, Grohvaz $F$, Valtorta $F$, Meldolesi I: Neurotransmitter release: fusion or 'kiss-and-run'. Trends Cell Biol 1994, 4:1-4.

24. Thomas P, Lee AK, Wong JG, Almers W: A triggered mechanism retrieves membrane in seconds after $\mathrm{Ca}^{2+}$-stimulated exocytosis in single pituitary cell. / Cell Biol 1994, 124:667-675.
25. Von Wedel RJ, Carlson SS, Larsson PA: Transfer of synaptic vesicle antigens to the presynaptic plasma membrane during exocytosis. Proc Natl Acad Sci USA 1981, 78:1014-1018.

26. Miller TM, Heuser JE: Endocytosis of synaptic vesicle membrane at the frog neuromuscular junction. I Cell Biol 1984 , 98:685-698.

27. Van der Blick AM, Meyerowitz EM: Dynamin-like protein encoded by the Drosophila shibire gene associated with vesicular traffic. Nature 1991, 351:411-414.

28. Chen MS, Obar RA, Schroeder CC, Austin TW, Poodry CA, Wadsworth SC, Valee RB: Multiple forms of dynamin are encoded by shibire, a Drosophila gene involved in endocytosis. Nature 1991, 361:583-586.

29. Kosaka T, Ikeda K: Reversable blockage of membrane retrieval and endocytosis in the garland cell of the temperature-sensitive mutant of Drosophila melanogaster, shibire. / Cell Biol 1983, 97:499-507.

30. Rosenboom $H$, Lindau $M$ : Exo-endocytosis and closing of the fission pore during endocytosis in single pituitary nerve terminals internally perfused with high calcium concentrations. Proc Natl Acad Sci USA 1994, 91:5267-5217.

31. Takei K, McPherson PS, Schmid SL, De Camilli P: Tubular - membrane invaginations coated by dynamin rings are induced by GTPS in nerve terminals. Nature 1995, 374:186-189.

It is shown that dynamin self-assembles into rings of the size seen around the necks of coated vesicles that fail to pinch off in shibire flies. In the presence of GTPYS, dynamin forms spirals around narrow tubules. It is suggested that dynamin may be a mechanochemical device that pinches vesicles off the cell surface, and provides the molecular basis for membrane fission. See also $\left[32^{\bullet \bullet}\right]$

32. Hinshaw JE, Schmid SL: Dynamin self-assembles into rings - suggesting a mechanism for coated vesicle budding. Nature $1995,374: 190-192$

See annotation $\left[31^{\bullet \bullet}\right]$

33. Monck JR, Fernandez IM: The exocytotic fusion pore and neurotransmitter release. Neuron 1994, 12:707-716.

34. Siegel DP: Modeling protein-induced fusion mechanisms: insights from the relative stability of lipic structures. In Viral fusion mechanisms. Edited by Bentz J. Boca Raton: CRC Press; 1993:475-512.

35. Neher E: Assymmetric membranes resulting from the fusion of two black lipid bilayers. Biochim Biophys Acta 1974, 373:327-336.

36. Hay $J C$, Fisette $P L$, Jenkins $G H$, Fukami $K$, Takenawa -. T, Anderson RA, Martin TFJ: ATP-dependent inositide phosphorylation required for $\mathrm{Ca}^{2+}$-activated secretion. Nature 1995, 374:173-177.

It is shown that type I phosphatidylinositol-5-kinase mediates one of the last ATP-requiring reactions in exocytosis in PC-12 cells, a neuroendocrine cell line. Given that a phosphatidylinositide transfer protein is also required for priming Ca-triggered exocytosis, it is concluded that the two enzymes in tandem create 4,5-polyphosphoinositides. The requirement for the two enzymes in exocytosis provides the most direct evidence yet that a specific lipid, 4,5-phosphatidylinositide, is required for exocytosis.

37. Pfenninger $K$, Akert $K$, Moor $H$, Sandri $C$ : The fine structure of freeze-fractured presynaptic membranes. I Neurocytol 1972, 1:129-149.

38. Zimmerberg J, Curran M, Cohen FS: A lipid/protein complex hypothesis for exocytotic fusion pore formation. Ann NY Acad Sci 1991, 635:307-317.

39. White $\mathrm{M}$ : Viral and cellular membrane fusion proteins. Annu Rev Physiol 1990, 52:675-697.

40. Bentz J, Ellens $H$, Alford D: An architecture for the fusion site of influenza hemagglutinin. FEBS Lett 1990, 276:1-5.

41. Bukauskas FF, Weingart R: Voltage-dependent gating of single gap junction channels in an insect cell line. Biophys / 1994, 67:613-625.

42. Bloom JA, Webb WW: Lipid diffusibility in the intact erythrocyte membrane. Biophys / 1983, 42:295-305. 
43. Oberhauser AF, Fernandez IM: Patch clamp studies of single intact secretory granules. Biophys J 1993, 65:1844-1852.

44. Monck JR, Alvarez de Toledo G, Fernandez JM: Tension in secretory granule membranes causes extensive membrane transfer through the exocytotic fusion pore. Proc Natl Acad Sci USA 1990, 87:7804-7808.

45. Hayashi T, McMahon $H$, Yamasaki S, Binz T, Hata $Y$, Südhof TC, Niemann H: Synaptic vesicle membrane fusion complex: action of clostridial neurotoxins on assembly. EMBO $/ 1994$. 13:5051-5061.

46. Thomas L, Hartung K, Longosch D, Rehm H, Bamberg E, Franke WW, Betz $\mathrm{H}$ : Identification of synaptophysin as a hexameric channel protein of the synaptic vesicle membrane. Science 1988, 242:1050-1053.

47. White IM: Membrane fusion. Science 1992, 258:917-936.

48. Wilson IA, Skehel JJ, Wilcy DC: Structure of the hemagglutinin membrane glycoprotein of influenza virus at $3 \dot{A}$ resolution. Nature 1981, 289:366-373.

49. Stegmann $T$, White JM, Helenius $A$ : Intermediates in influenza induced membrane fusion. EMBO / 1990, 9:4231-4241.

50. Stegmann T, Delfino JM, Richards FM, Helenius A: The HA2 subunit of influenza hemagglutinin inserts into the target membrane prior to fusion. I Biol Chem 1991, 266:18404-18410.

51. Tsurudome $M$, Glück $R$, Graf $R$, falchetto $R$, Schaller $U$, Brunner J: Lipid interaction of the hemagglutinin HA2 NH2-terminal segment during influenza virus-induced membrane fusion. I Biol Chem 1992, 267:20225-20232.

52. Harter $C$, James $P$, Bächi T, Semenza $C$, Brunner ): Hydrophobic binding of the ectodomain of influenza hemagglutinin to membranes occurs through the fusion peptide. I Biol Chem 1989, 264:6459-6464.

53. Carr C, Kim PS: A spring-loaded mechanism for the conformational change of influenza hemagglutinin. Cell 1993, 73:823-832

54. Bullough PA, Hughson FM, Skehel J), Wiley DC: Structure of influenza haemagglutinin at the $\mathrm{pH}$ of membrane fusion. Nature 1994, 371:37-43.

First crystal structure of a portion of influenza haemagglutinin (HA) at acidic pH. HA is physiologically cleaved into two subunits that remain connected by disulfide bonds; HAO contains the sialic acid binding site and HAl the fusion peptide. A thermolysin fragment of HAI was generated; it lacked the transmembrane domain and the fusion peptide, did not, therefore, aggregate in solution, and formed crystals.
55. Sölner $T$, Whiteheart $S$, Brunner $M$, Erdjument-Bromage $H$, Ceromanos $M$, Tempst $P$, Rothman JE: SNAP receptors implicated in vesicle targeting and fusion. Nature 1993, 362:318-323.

56. Tse FW, Iwata A, Almers W: Membrane flux through the pore formed by a fusogenic viral envelope protein during cell fusion. J Cell Biol 1993, 121:543-552.

57. Zimmerberg I, Blumenthal R, Sarkar DP, Curran M, Morris S): Restricted movement of lipid and aqueous dyes through pores formed by influenza hemagglutinin during cell fusion. $j \mathrm{Cell}$ Biol 1994, 127:1885-1894.

58. Kemble GW, Danieli T, White JM: Lipid-anchored influenza - hemagglutinin promotes hemifusion, not complete fusion. Cell 1994, 76:383-391.

The transmembrane domains of influenza haemagglutinin (HA) were replaced with glycophosphatidylinositol anchors and expressed in Chinese hamster ovary $(\mathrm{CHO})$ cells. Fluorescently labeled erythrocytes (RBCs) were attached to the $\mathrm{CHO}$ cells. After acidification, the transfer of fusing membranes occured at normal rates, but soluble fluorescent dye enclosed in the RBC failed to diffuse into the CHO cells. The paper proves that HA cannol catalyze fusion without its transmembrane domains.

59. Chow RH, Von Lüden L, Neher E: Delay in vesicle fusion revealed by electrochemical monitoring of secretory events in adrenal chromaffin cells. Nature 1992, 356:60-63.

60. Ureña I, Fernández-Chacón R, Benot AR, Alvarez de Toledo $\mathrm{G}$, López-Barneo J: Hypoxia induces voltage-dependent $\mathrm{Ca}^{2+}$ entry and quantal dopamine secretion in carotid body glomus cells. Proc Natl Acad Sci USA 1994, 91:10208-10211.

61. Girod R, Correges $P$, Jacquet J, Dunant $Y$ : Space and time characteristics of transmitter release at the nerve-electroplaque junction of Torpedo marmorata. J Physiol 1993, 471:129-157.

62. Stegmann T: Anchors aweigh. Curr Biol 1994, 4:551-554

63. Hughson FM: Structural characterization of viral fusion proteins. Curr Biol 1995, 5:265-274.

64. Lanzrein $M$, Käsermann $R$, Weingart $R$, Kempf $C$ : Early events of semliki forest virus-induced cell-cell fusion. Virology 196:541-547.

M Lindau and W Almers, Abteilung Molekulare Zellforschung, Max Planck Institut für medizinische Forschung, Jahnstrasse 19, 69120 Heidelberg, Germany. 